\title{
3 Research Square

\section{The Serum Level of Vitamin D and Hypocalcemia in Thyroidectomy Candidate Patients Referred to a Tertiary Care Hospital in the North of Iran}

Hossein Hemmati ( $\boldsymbol{D}$ drhossein.hemmati@gmail.com )

Mehdi Poursafar

Guilan University of Medical Sciences

Mohaya Farzin

Guilan University of Medical Sciences

Behrang Motamed

Guilan University of Medical Sciences

Pirooz Samidoost

Guilan University of Medical Sciences

\section{Elahe Rafiei}

Guilan University of Medical Sciences

Soudabeh Bahush

Guilan University of Medical Sciences

\section{Research note}

Keywords: Postoperative hypocalcemia, total thyroidectomy, vitamin D deficiency

Posted Date: July 2nd, 2020

DOI: https://doi.org/10.21203/rs.3.rs-38880/v1

License: (c) (i) This work is licensed under a Creative Commons Attribution 4.0 International License.

Read Full License 


\section{Abstract}

Objective: Vitamin D plays a crucial role in calcium metabolism through the Parathyroid hormone (PTH) dependent process. The deficiency of this important nutrient may be associated with hypocalcemia after thyroidectomy. To evaluate the role of vitamin $\mathrm{D}$ in predicting hypocalcemia following total thyroidectomy.

Results: sixty-two patients who underwent total thyroidectomy for benign or malignant thyroid disease were included in this prospective study. Preoperative vitamin D serum levels and PTH levels were determined. The results of our study showed In both groups with and without vitamin D deficiency, calcium levels decreased significantly $(P=0.01)$. In our study, it was found that there was no significant relationship between postoperative hypocalcemia and vitamin $D$ deficiency. $(P=0.441)$. After reviewing the data and according to Spearman correlation statistical test, no significant relationship was observed between serum PTH and calcium after thyroidectomy $(P=0.340)$.Vitamin $D$ deficiency is a risk factor of hypocalcemia after total thyroidectomy for benign goiter. Although post thyroidectomy hypocalcemia is multifactorial, vitamin D deficiency, particularly severe form, is significantly associated with the development of biochemical and clinical hypocalcemia vitamin $D$ supplementation can prevent this unwanted complication in such patients.

\section{Introduction}

Total thyroidectomy is one of the most performed surgical procedures worldwide [1].Total thyroidectomy (TT) is a routine for the surgical treatment of multinodular goiter, while postoperative hypocalcemia is the frequent complication. The etiology of postoperative hypocalcemia is multifactorial, even though the most important component is surgical trauma to the parathyroid glands [2].It is frequently mild and transitory (in up to $50 \%$ of cases) and infrequently permanent (5\%) [3]. Nevertheless, it is constantly concern and challenge for surgeons, and patients [4]. TT might cause hypoparathyroidism due to the ignored removal of the parathyroid gland or damage to its blood supply [5]. It has been experimental that vitamin $D$ deficiency favoritisms the entrance of postoperative hypocalcemia in patients who undergo surgery for multinodular goiter or Graves'-Basedow disease [6]. Vitamin D shows a central role in the homeostasis of calcium and the intestinal absorption of calcium and phosphate [7]. However, the role of vitamin $\mathrm{D}$ in the prediction of postoperative hypocalcemia still remnants a provocative issue [8].

The synthesizes its active form in the kidneys (1-25[0H]D3) finished a process controlled by parathyroid hormone (PTH), and therefore by increasing vitamin D levels, the secretion of PTH is reduced [9]. If this connection between preoperative vitamin D deficiency and postoperative hypocalcemia is confirmed, vitamin $D$ levels might be a decent indicator of this risk that could be tranquil to the quantity and correct before surgery [10].

Due to the role of vitamin $\mathrm{D}$ in calcium metabolism in the body and the existence of few studies on the relationship between vitamin $\mathrm{D}$ levels and calcium levels after thyroidectomy, we decided to conduct a 
study to determine the level of vitamin $D$ in patients with thyroidectomy and its effect on calcium and hypocalcemia after thyroidectomy. In this study, an attempt was made to closely monitor patients' preand post-hospitalization, inpatient and preoperative, during, postoperative, and postoperative interventions to reduce the involvement of confounding factors in the results. Measure vitamin D with a higher level of calcium and possible hypocalcemia with higher accuracy and provide a more conclusive result for practical purposes.

\section{Methods}

\section{Participants}

A retrospective study conducted on a group of patients who underwent total and subtotal thyroidectomy in 2018-2020 referred to Razi hospital in the north of Iran. The study was performed by the declaration of Helsinki and approved by the Ethics Committee of Guilan University of Medical Sciences (IR.GUMS.REC.1397.463). Inclusion criteria were indications for thyroidectomy (included any kind or suspicious mass or malignancies that had previously been confirmed by a pathology examination). Exclusion criteria were preoperative hypocalcemia, parathyroid adenoma, any underlying disease that affects parathyroid hormone levels, vitamin D, calcium, and albumin (such as chronic kidney disease, indigestion, and malabsorption), patient dissatisfaction participating in the study included a previous major procedure such as total laryngectomy or parathyroidectomy, receiving prophylactic drug therapy for osteoporosis, including calcium and vitamin D supplementation, and patients with hyperthyroidism and active Grave's.

\section{Experimental design}

Based on vitamin D levels before surgery, patients were divided into two groups with normal vitamin $D$ (between 30-30 ng/ml) and vitamin D deficiency (less than $30 \mathrm{ng} / \mathrm{ml}$ ). Then, postoperative calcium changes in patients were measured in two forms: normal calcium $(8.5-5.5 \mathrm{mg} / \mathrm{dl}$ ) and hypocalcemia (less than $8.5 \mathrm{mg} / \mathrm{dl}$ ) based on vitamin D levels before surgery. Calcium, phosphorus, parathormone, and vitamin D levels were measured by the enzyme-linked immunosorbent assay (ELISA). Biotek microplate reader ELX-800, made in 750-400 nm microplates, has 4 filters, 630, 9090, $405 \mathrm{~nm}$, source Tungsten light, and resolution are $0.001 \mathrm{OD}$. The status of thyroid tissue cytopathology was also calculated based on a biopsy performed according to the Bethesda standard. Based on this, the patient's pathology will be divided into 6 categories. Accordingly to (I) non-diagnostic; (ii) benign; (iii) an atypically insignificant atypia (AUS) or an unexplained follicular lesion (FLUS); (iv) Follicular neoplasm or suspected follicular neoplasm; (V) Suspected of malignancy and (vi) malignant. The information of all patients who entered the study, including age, sex, underlying disease, body mass index, length of hospital stay, type of thyroid pathology, serum calcium levels, phosphorus, PTH before and immediately after surgery, and vitamin D levels before surgery were measured and It was recorded in the pre-prepared information form. The volume of the sample required to evaluate the serum level of vitamin D before surgery with hypocalcemia after thyroidectomy with $95 \%$ confidence (significance level $5 \%$ ) and $90 \%$ test power based on the results 
of Tripathi et al.'s study [11] according to the minimum sampling formula 31 people were estimated in each group.

\section{Data analysis and statistics}

The SPSS software version 21.0 was used for data analyses. To describe quantitative variables with normal distribution, mean and standard deviation (about 95\% confidence) were used, and for quantitative variables with abnormal distribution, the mean and intermediate-range were used. Qualitative variables were also described based on numbers and percentages. Normal distribution of quantitative study variables was measured using tensile and skew values, and Shapiro-Wilk test. To compare quantitative variables between two groups with and without vitamin D deficiency from the independent t-test; and covariance analysis; ANCOVA was used by modifying the variable effect before surgery. Comparison of quantitative variables before and after thyroidectomy using paired t-test; paired t-test (equivalent to nonparametric Wilcoxon) was used. To compare hypoxia in two groups, the Chi-Square test and, if not validated, the Fisher's Exact Test. Correlation of variables using Pearson correlation coefficient; Pearson correlation (Spearman correlation) was measured.

\section{Results}

A total of 62 patients with a mean age of $47 \pm 18.9$ years were included in the study. Of these patients, 53 (85.5\%) were female and 9 (14.5\%) were male. There were no significant differences based on age mean and gender between two groups of patients with normal vitamin $D$ and vitamin D deficiency. Regarding the underlying disease, 43 patients (69.4\%) did not have an underlying disease, 19 patients $(30.7 \%)$ had one to three underlying diseases. Of these, high blood pressure with $7(11.3 \%)$ cases had the highest prevalence. The Body mass index (BMI) of patients was $35.6 \pm 18.29$. After examining the data and using the Mann Whitney test, there was no significant difference between the BMI status in the two groups $(P=0.642)$ (Table 1). Among the patients assessed, pathology findings were not significantly different in the two groups $(P=0.980)$ (Table 2$)$.

The incidence of symptomatic hypocalcemia was $(n=32)$ and asymptomatic hypocalcemia (only the laboratory hypocalcemia; $\mathrm{Ca}<8 \mathrm{mg} / \mathrm{dl})$ was $(\mathrm{n}=30)$. the mean of serum calcium after thyroidectomy decreased in both vitamin $D$ studied groups but these differences were not statistically significant in any of these groups ( $p>0.05)$. Incidence of hypocalcemia in two groups with and without vitamin $D$ deficiency was $56.7 \%(\mathrm{~N}=17)$ and $46.3 \%(\mathrm{~N}=13)$, there was not any association between postoperative hypocalcemia and vitamin $D$ deficiency $(p>0.05)$.

In the present study, between calcium and parathyroid hormone after surgery in two groups with and without vitamin $D$ deficiency, no significant correlation was obtained. $(P=0.340)$. There was no significant association between postoperative phosphorus and postoperative parathyroid hormone in the deficiency of the vitamin $D$ group. $(p>0.05)$ The association between postoperative phosphorus and postoperative parathyroid hormone was not significantly associated with vitamin $D$ deficiency $(p>0.05)$. A significant difference was observed in the vitamin $D$ deficiency group in the comparison between 
postoperative parathyroid hormone and preoperative parathyroid hormone $(P=0.045)$. Comparison of postoperative parathyroid hormone and preoperative parathyroid hormone in the group without vitamin $D$ deficiency there was a significant difference. $(P=0.006)$

Also, no significant difference was observed in the group without vitamin $D$ deficiency compared to postoperative phosphorus and preoperative phosphorus $(P=0.125)$. There was no significant difference in vitamin $D$ deficiency between preoperative and postoperative phosphorus deficiency $(P=0.0784)$.

\section{Discussion}

Vitamin D deficiency has emerged as a significant public health problem throughout the world [12]. Although hypocalcemia is a common complication following thyroidectomy, it is usually temporary, and only $1-3 \%$ of the patients develop enduring hypocalcemia which is distinct as ongoing hypocalcemia within 6 months after surgery [13].Vitamin D is the principal factor that maintains calcium homeostasis [14]. The results of our study showed that factors such as age, sex, BMI, length of hospital stay, and underlying thyroid pathology did not significantly different between the two groups, so these underlying factors did not lead to bias in our patients. Studies are showing that transient hypocalcemia is associated with aging $[15,16]$ A systematic study by Edafe et al. showed that the mean age of patients with transient hypocalcemia and those who did not have significant differences were observed. Also, some studies have reported more postmenopausal hypocalcemia in women [17]. As mentioned, there was no significant difference between age and sex in our study groups, so these two factors were not a factor in the study of hypocalcemia in the two groups under study. Some studies, such as a study by Kirkby-B et al. Of hypocalcemia in patients, were associated with an increased length of hospital stay [18] .In our study, because radiation therapy was similar in our patients after thyroidectomy, the effect of hospital stay time as a possible cause of calcium change was removed from the disruptive factors. Our patients were divided into two groups with vitamin D deficiency and adequate vitamin D levels. Calcium, phosphorus, and PTH were assessed before and after surgery in these patients. The results of our study

showed that there was no significant association between postoperative hypocalcemia and postoperative vitamin D deficiency, and both groups studied in our study (vitamin D deficiency and adequate vitamin D) showed moderate to moderate calcium deviation. Vitamin D plays an important role in calcium metabolism by increasing calcium absorption for the gastrointestinal tract [19] The results of our study were similar to those of Chia et al [20].According to the results of our study, serum levels of vitamin D before complete thyroidectomy do not play a role in postoperative hypocalcemia, which is one of the most important and approximately common complications of this surgery. In the last two decades, total thyroidectomy has become one of the treatment options for malignant and benign thyroid diseases, leading to post-thyroid hypocalcemia [21]. In our study, there was no significant association between serum PTH and calcium levels in the two groups, but PTH before and after surgery showed significant differences between the two groups, but between phosphorus before and after surgery in the two groups. You haven't seen it. Some studies have suggested that measuring PTH on the first day after surgery may be a useful method for predicting hypocalcemia after a thyroidectomy, thus preventing long-term hospitalization. In addition, the PTH dose on the first day after surgery is more reliable and cheaper than 
the rapid PTH procedure during surgery [22]. The strengths of this study include the use of a forwardlooking database to extract large amounts of data, including the symptoms of hypocalcemia, calcium replacement, and long-term hypoparathyroidism. The uniformity of surgery and the performance of a surgeon are other strengths of our study. This hypothesis requires further research to determine effective parameters in the future. Vitamin D deficiency was common in our population of patients undergoing thyroidectomy; however, it was not associated with an increased risk of postoperative hypocalcemia. According to the results of our study, serum levels of vitamin $D$ before thyroidectomy do not play a role in postoperative hypocalcemia, which is one of the most important and approximately common complications of this surgery. Careful and prolonged follow-up of calcium, vitamin $\mathrm{D}$, and parathyroid hormone levels should be suggested for these patients. Therefore, preoperative oral supplementation of vitamin $\mathrm{D}$ should be considered, to minimize this risk. Therefore, preoperative oral supplementation of vitamin $\mathrm{D}$ could be considered, to minimize this risk.

\section{Limitations}

Further studies are needed with larger number of the participants to clarify the serum level of vitamin $D$ and hypocalcemia in thyroidectomy. Moreover, the results limited to single center in the north of country and can not represent the general population.

\section{Abbreviations}

PTH: Parathyroid hormone; TT: Total thyroidectomy; ELISA: enzyme-linked immunosorbent assay; BMI: Body mass index

\section{Declarations}

\section{Authors' contributions}

$\mathrm{HH}, \mathrm{PS}, \mathrm{BM}, \mathrm{ER}$ and MP conceived and designed the experiments and read and approved the final manuscript. HH, MP, MF, BM, PS, and SB implemented and conducted the experiments. ER, SB, and MF mainly analyzed the data and wrote the manuscript. All authors read an approved the final manuscript.

\section{Acknowledgments}

We are especially grateful to all the experts who were integral partner in the preparation of facilities.

\section{Conflict of interest}

The authors declare that they have no conflict of interest concerning this article.

Funding: This study was supported financially by the research council from Guilan University of Medical Sciences [grant number 1074]. 


\section{Availability of data and materials}

Data will not be shared and available for authors.

\section{Ethics approval and consent to participate}

All procedures used in this study were in accordance with the Declaration of Helsinki and approved by the Ethics Committee at Guilan of Medical Sciences University (No. 97112705). The participants gave written informed consent for the study after receiving a detailed explanation of the purposes, potential benefits, and risks associated with participation.

\section{Consent for publication}

Not applicable. The study design was retrospective and only medical records were used. All procedures was performed as a part of routine healthcare services for hospitalized patients.

\section{Competing interests}

The authors declare that they have no competing interests.

\section{References}

1. Khanzada TW, Samad A, Memon W, Kumar B. Post thyroidectomy complications: the Hyderabad experience. Journal Ayub Medical College Abbottabad. 2010;22(1):65-8.

2. Dralle H, Lorenz K, Machens A. State of the art: surgery for endemic goiter-a plea for individualizing the extent of resection instead of heading for routine total thyroidectomy. Langenbeck's archives surg. 2011;396(8):1137-43.

3. Hankey GJ, Warlow CP, Sellar RJ. Cerebral angiographic risk in mild cerebrovascular disease. Stroke. 1990;21(2):209-22.

4. Say RE, Thomson R. The importance of patient preferences in treatment decisions-challenges for doctors. BMJ. 2003;327(7414):542-5.

5. Jung SY, Kim HY, Park HS, Yin XY, Chung SM, Kim HS. Standardization of a physiologic hypoparathyroidism animal model. Plos one. 2016;11(10):e0163911.

6. Kim WW, Chung S-H, Ban EJ, Lee CR, Kang S-W, Jeong JJ, et al. Is preoperative Vitamin D deficiency a risk factor for postoperative symptomatic hypocalcemia in thyroid cancer patients undergoing total thyroidectomy plus central compartment neck dissection? Thyroid. 2015;25(8):911-8.

7. Veldurthy V, Wei R, Oz L, Dhawan P, Jeon YH, Christakos S. Vitamin D, calcium homeostasis and aging. Bone rese. 2016;4:16041-.

8. Schmidt-Gayk H, Armbruster FP, Bouillon R. Calcium regulating hormones, vitamin D metabolites, and cyclic AMP assays and their clinical application: Springer Science \& Business Media; 2012. 
9. Still CD, Benotti P, Hangan D, Zubair F. Metabolic Complications, Nutritional Deficiencies, and Medication Management Following Metabolic Surgery. Complications Bariatric Surg: Springer; 2018. p. 5-33.

10. Sommers MS, Fannin E. Diseases and disorders: A nursing therapeutics manual: FA Davis; 2014.

11. Tripathi M, Karwasra RK, Parshad S. Effect of preoperative vitamin D deficiency on postoperative hypocalcemia after thyroid surgery. Thyroid Res. 2014;7:8-.

12. Tripathi M, Karwasra RK, Parshad S. Effect of preoperative vitamin D deficiency on postoperative hypocalcemia after thyroid surgery. Thyroid Res. 2014;7(1):8.

13. Holmøy T, Moen S. Assessing vitamin D in the central nervous system. Acta Neurologica Scandinavica. 2010;122:88-92.

14. Langeveld $\mathrm{H}$, Weidema W, Stassen L, Bonjer $\mathrm{H}$, Steyerberg $\mathrm{E}$, Lange J, et al. 15 th International Congress of the European Association for Endoscopic Surgery (EAES) Athens, Greece, 4-7 July 2007 Oral presentations. Surg Endosc. 2008;22:S1-S47.

15. Veldurthy V, Wei R, Oz L, Dhawan P, Jeon YH, Christakos S. Vitamin D, calcium homeostasis and aging. Bone rese. 2016;4(1):1-7.

16. Edafe O, Antakia R, Laskar N, Uttley L, Balasubramanian S. Systematic review and meta-analysis of predictors of post-thyroidectomy hypocalcaemia. British Journal Surg. 2014;101(4):307-20.

17. Ozogul B, Akcay MN, Akcay G, Bulut OH. Factors affecting hypocalcaemia following total thyroidectomy: a prospective study. Eurasian J Med. 2014;46(1):15.

18. Edafe O, Antakia R, Laskar N, Uttley L, Balasubramanian S. Systematic review and meta-analysis of predictors of post-thyroidectomy hypocalcaemia. British Journal Surg. 2014;101(4):307-20.

19. Godazandeh G, Kashi Z, Godazandeh F, Tayebi P, Bijani A. Influence of thyroidectomy on postoperative serum calcium level regarding serum vitamin D status. A prospective study. Caspian J Intern Med. 2015;6(2):72-6.

20. Kirkby-Bott J, Markogiannakis H, Skandarajah A, Cowan M, Fleming B, Palazzo F. Preoperative vitamin $D$ deficiency predicts postoperative hypocalcemia after total thyroidectomy. WORLD J SURG. 2011;35(2):324-30.

21. Al-Khatib T, Althubaiti AM, Althubaiti A, Mosli HH, Alwasiah RO, Badawood LM. Severe vitamin D deficiency: a significant predictor of early hypocalcemia after total thyroidectomy. Otolaryng Head Neck. 2015;152(3):424-31.

22. Chia SH, Weisman RA, Tieu D, Kelly C, Dillmann WH, Orloff LA. Prospective study of perioperative factors predicting hypocalcemia after thyroid and parathyroid surgery. Archives of OtolaryngologyHead \& Neck Surg. 2006;132(1):41-5.

\section{Tables}


Table 1: Patient characteristics and clinical data of the study population

\begin{tabular}{|c|c|c|c|c|c|}
\hline \multirow[t]{3}{*}{ Variable } & \multicolumn{5}{|c|}{ Cases } \\
\hline & \multicolumn{2}{|c|}{ Valid } & Missing & \multicolumn{2}{|l|}{ Total } \\
\hline & $\mathrm{N}$ & Percent & Percent & $\mathrm{N}$ & Percent \\
\hline Age & 62 & $100.0 \%$ & $0.0 \%$ & 62 & $100.0 \%$ \\
\hline Age Insufficient & 32 & $100.0 \%$ & $0.0 \%$ & 32 & $100.0 \%$ \\
\hline Age Sufficient & 30 & $100.0 \%$ & $0.0 \%$ & 30 & $100.0 \%$ \\
\hline Sex & \multicolumn{2}{|c|}{ Frequency } & Percent & $\begin{array}{l}\text { Valid } \\
\text { Percent }\end{array}$ & $\begin{array}{l}\text { Cumulative } \\
\text { Percent }\end{array}$ \\
\hline Male & \multicolumn{2}{|l|}{9} & 14.5 & 14.5 & 14.5 \\
\hline Female & \multicolumn{2}{|l|}{53} & 85.5 & 85.5 & 100.0 \\
\hline Total & \multicolumn{2}{|l|}{62} & 100.0 & \multicolumn{2}{|l|}{100.0} \\
\hline $\begin{array}{l}\text { Underlying } \\
\text { Diseases }\end{array}$ & \multicolumn{2}{|l|}{43} & 69.4 & 69.4 & 69.4 \\
\hline DM & \multicolumn{2}{|l|}{3} & 4.8 & 4.8 & 74.2 \\
\hline HTN & \multicolumn{2}{|l|}{7} & 11.3 & 11.3 & 85.5 \\
\hline HLP & \multicolumn{2}{|l|}{2} & 3.2 & 3.2 & 88.7 \\
\hline DM HTN & \multicolumn{2}{|l|}{3} & 4.8 & 4.8 & 93.5 \\
\hline DM HLP & \multicolumn{2}{|l|}{1} & 1.6 & 1.6 & 95.2 \\
\hline HTN HLP & \multicolumn{2}{|l|}{2} & 3.2 & 3.2 & 98.4 \\
\hline DM HTN HLP & \multicolumn{2}{|l|}{1} & 1.6 & 1.6 & 100.0 \\
\hline \multirow[t]{2}{*}{ Total } & \multicolumn{2}{|l|}{62} & 100.0 & 100.0 & \\
\hline & & & & Statistic & Std. Error \\
\hline Body Mass Index & & & & 29.1834 & .80679 \\
\hline & & Confidence Interval for & Lower Bound & 27.5701 & \\
\hline & & & Upper Bound & 30.7967 & \\
\hline & & rimmed Mean & & 28.4071 & \\
\hline & & & & 27.0552 & \\
\hline & & ince & & 40.357 & \\
\hline
\end{tabular}


Table 2: Abundant distribution of pathological status in two groups with and without Vitamin D

\begin{tabular}{|c|c|c|c|c|}
\hline \multirow[t]{2}{*}{ Pathology } & & \multicolumn{2}{|l|}{ Vit D } & \multirow[t]{2}{*}{ Total } \\
\hline & & Insufficient & Sufficient & \\
\hline Benign & $\begin{array}{l}\% \text { within } \\
\text { keifi }\end{array}$ & $31.3 \%$ & $33.3 \%$ & $32.3 \%$ \\
\hline AUS/FLUS & $\begin{array}{l}\% \text { within } \\
\text { keifi }\end{array}$ & $15.6 \%$ & $13.3 \%$ & $14.5 \%$ \\
\hline $\begin{array}{l}\text { Ferrochloric uptake / or suspected follicular } \\
\text { neoplasm }\end{array}$ & $\begin{array}{l}\% \text { within } \\
\text { keifi }\end{array}$ & $6.3 \%$ & $6.7 \%$ & $6.5 \%$ \\
\hline Suspicious of malignancy & $\begin{array}{l}\% \text { within } \\
\text { keifi }\end{array}$ & $15.6 \%$ & $23.3 \%$ & $19.4 \%$ \\
\hline Malignant & $\begin{array}{l}\% \text { within } \\
\text { keifi }\end{array}$ & $12.5 \%$ & $13.3 \%$ & $12.9 \%$ \\
\hline Multi-nodular goiter & $\begin{array}{l}\% \text { within } \\
\text { keifi }\end{array}$ & $15.6 \%$ & $10.0 \%$ & $12.9 \%$ \\
\hline sympathetic guitar & $\begin{array}{l}\% \text { within } \\
\text { keifi }\end{array}$ & $3.1 \%$ & $0.0 \%$ & $1.6 \%$ \\
\hline Total & $\begin{array}{l}\% \text { within } \\
\text { keifi }\end{array}$ & $100.0 \%$ & $100.0 \%$ & $100.0 \%$ \\
\hline
\end{tabular}

